ORIGINAL ARTICLE

\title{
The Treatment Results in Patients with Aluminum Phosphide Toxicity Admitted to Razi Hospital in Ahvaz
}

\author{
FARKHONDEH JAMSHIDI ${ }^{1}$, MALIKA VAEZ TEHRANI ${ }^{2}$, AHMAD GHORBANI $^{3}$ \\ ${ }^{1}$ Department of Forensic Medicine and Toxicology, Ahvaz Jundishapur University of Medical Sciences, Ahvaz, Iran \\ ${ }^{2}$ Student Research Committee, Ahvaz Jundishapur University of Medical Sciences, Ahvaz, Iran. \\ ${ }^{3}$ Department of Forensic Medicine and Toxicology, Ahvaz Jundishapur University of Medical Sciences, Ahvaz,Iran. \\ Correspondence to: Malika Vaez Tehrani, Student Research Committee, Ahvaz Jundishapur University of Medical Sciences, Ahvaz, Iran. \\ Tel: +981131156704
}

\begin{abstract}
Introduction: Aluminum phosphide (ALP), locally known as "Rice Tablet", is a cheap and powerful pesticide used frequently to protect cereals. Cases of ALP toxicity have grown dramatically in recent decades by using it for suicide purposes. Treatment of ALP toxicity is largely supported by various remedies as there is no specific antidote. This study deals with the epidemiology of ALP toxicity in Khuzestan (Iran) and assesses the clinical and paraclinical manifestations, as well as the results of various treatments during the last 12 years. The study further examines the next suicidal intentions and subsequent complications of poisoning with ALP.

Methodology: The records of all patients with ALP toxicity admitted to Razi Hospital in Ahvaz from 2006 to 2017 were reviewed within a retrospective study. Epidemiological and demographic information, clinical and paraclinical manifestations, and the results of various treatments were extracted. Data on the subsequent complications and the next suicidal intentions in those who have survived a suicide attempt were gathered and analyzed.

Results: Of the 48 patients studied, $50 \%$ were male and the mean age was $29.33 \pm 10.45$. Most of the toxicities were orally and with the intention of suicide in Ahvaz and Andimeshk. A total of $71.42 \%$ of the dead have been carried to this center. The majority of cases were reported from urban areas and in August and during summer. The most prevalent clinical signs were loss of consciousness, nausea, and vomiting. There were some differences in the experimental and EKG results. A meaningful correlation was observed between the administration and/or consumption of ranitidine, NAC, and intravenous sodium bicarbonate with treatment outcomes. Only one case of reattempt to suicide has been reported, and hematuria, ascites, and pleural effusion were widely visible in survivors.

Conclusion: On-call counseling may decrease the rate of mortality in dispatching patients. Clinical and paraclinical manifestations can be useful in the diagnosis or prognosis. Patients' follow-up, actions to enhance public awareness, restrictions on the distribution of ALP, and availability of alternative substances can be helpful to diminish cases of ALP toxicity and/or death.
\end{abstract}

Keywords: Aluminum phosphide (ALP), Rice tablets, Poisoning, Khuzestan

\section{INTRODUCTION}

Metal phosphides (aluminum, zinc, magnesium, and calcium) have long been accepted as rodenticides and pesticides across the world [1]. Low cost, high strength in killing harmful insects and rodents, leaving no toxic residues, and no adverse consequences on the health of grains are factors contributing to the extensive application of metal phosphides[2]. These compounds of phosphorus are widely utilized in all stages of transportation of cereals by ships or trains (for example) and their storage in silos. These compounds release phosphine gas $(\mathrm{PH} 3)$ upon contact with moisture[3].

Aluminum phosphide breaks into $\mathrm{PH} 3$ and aluminum hydroxide in the presence of water. The release of $\mathrm{PH} 3$ will be more severe if the tablet comes in contact with an acidic solution such as hydrochloric acid [4]. Normally, upon swallowing, metal phosphides react with the acidic juice in the gastrointestinal tract, resulting in rapid phosphine release and absorption, as well as inhibition of the activity of enzyme esterase[5].

Among early symptoms, the garlic smell can be recognized from the patients' breath as a result of toxicity with phosphides[6]. Other clinical symptoms are dosedependent, appeared over time, and detected with diagnostic tests. Symptoms of toxicity appear rapidly upon consumption, i.e., within 10-15 minutes after swallowing.
Recent studies showed that some toxins induce hemolysis[7]. In patients with mild poisoning, nausea, recurrent vomiting, diarrhea, abdominal pain, and discomfort, especially in the epigastric region and tachycardia, are common[8].

Moderate to severe poisoning will appear rapidly into gastrointestinal manifestations, refractory hypotension and shock, palpitations, cardiovascular collapse, dysrhythmia, tachypnea, and ARDS. Restlessness, anxiety, ataxia, paraesthesia, and tremor are visible in all patients. A secondary condition (e.g., hypoxia) intensifies central neurological manifestations such as delirium, seizures, and coma [9].

\section{MATERIAL AND METHOD}

Type of study: A retrospective, applied, descriptive study was carried out in 2018 to evaluate the treatment results in patients with aluminum phosphide toxicity admitted to the Razi Hospital of Ahvaz Jundishapur University of Medical Sciences, a Clinical Toxicology Center located in Southwest Iran.

The results obtained from this study can be useful in healing patients with ALP poisoning and adopting health policies.

Sampling: Data were gathered by the census in which the files of all patients who were admitted to Razi Hospital in 
Ahvaz with the final diagnosis of ALP toxicity, from April 1, 2006, to the end of March 2017, were reviewed.

Data analysis: Data of qualitative variables were analyzed by frequency and percentage and that of quantitative variables with mean and standard deviation, as well as, median and quartile deviation (QD) if required.

The one-way analysis of variance (ANOVA), independent t-test, and Pearson correlation, and if required, their non-parametric equivalent tests (i.e., Kruskal-Wallis, Mann-Whitney, and Spearman correlation) were used to examine the univariate relationship between survival status with other variables. Multivariate regression and, if needed, logistic regression were employed coincidently.

All data were analyzed in IBM SPSS Statistics v22.

\section{DISCUSSION AND RESULT}

Epidemiology and demography: A total of $50 \%$ of patients were male and the mean age of all participants was $29.33 \pm 10.45$. There was a consistency between the mean age in this study with those reported in other studies[8], which may be due to the tendency of young adults in using this compound for suicide[10]. There was no meaningful correlation between the gender and age of participants and treatment outcomes.

A total of $83.3 \%$ of patients were born in Khuzestan and $93.8 \%$ were inhabitants of this province. Most patients were citizens of Ahvaz and its outskirts, following by Behbahan and Andimeshk.

A total of $85.4 \%$ of toxicities included oral poisoning with suicidal intentions.

The majority of cases of oral intake for suicide have been reported from Ahvaz and its suburbs, Andimeshk, Behbahan, Izeh, and Shush, respectively.

A total of $8.33 \%$ of toxicities were by inhalation. Inhalation toxicity still seems to be rare and due to the unwanted presence in an environment where phosphine gas is emitted from the solid form of the substance [11].

The prevalence of ALP toxicity has been higher in the rural $(81.7 \%)$, in compliance with the results of other recent studies[12]. However, there was no meaningful association between being rural and urban and the outcome of treatment. The highest number of hospitalizations (43.75\%) was between 18:00 and 24:00.

The highest number of oral ALP consumption for suicidal intentions (14.6\%) was in August, and no toxicity has been recorded in April.

The prevalence of toxicity in summer $(29.2 \%)$ was higher than in other seasons. This finding is inconsistent with the results of other studies[13], probably due to climatic differences.

The toxicity-caused lethality has increased over the past twelve years, and such increase is consistent with figures reported in other studies[6]. The rate of mortalities was highest in patients admitted in hospitals in Ahvaz, following Andimeshk and Izeh.

There was no meaningful difference in the number of tablets taken between rescued $(1.8 \pm 2.9)$ and dead (1.6 \pm 0.71$)$ patients. This suggests that other factors, such as the time and treatment plan, are more important than the dose of toxin in toxicity.
The number of tablets taken in the rescued patients was not associated with the length of hospitalization or ICU admission.

The mean length of hospitalization of rescued patients was $2.91 \pm 1.31$ days. The corresponding figure for dead patients was $11 \pm 9.13$ hours with a median of 11.5 hours, indicating that although some patients are admitted in advanced stages with a severe and pre-arrest condition, in most cases, the nature of the toxicity and the timing allow prescribing proper treatments modulating and alleviating risk factors such as acidosis.

A total of $71.42 \%$ of deaths were among dispatched patients. In general, there is no meaningful relationship between dispatching and death. However, given the high percentage of dead patients from dispatched ones, confounding factors seem to be effective in investigating the relationship between dispatching and death. For example, reviewing the files of patients who have been directly undergone CPR or patients who have been admitted after the risk alleviation shows that more principled treatment in the primary centers increases the possibility of rescuing the patient[14].

Clinical manifestations: Nausea and vomiting, abdominal pain, dizziness and headache, epigastric pain, thirst and weakness, and lethargy were the most common conditions reported by patients, which are consistent with other studies. Among these conditions, a meaningful association was observed between weakness and lethargy with death.

For physical examinations, loss of consciousness (lethargy, agitation, or coma), ill and toxicity, weak pulse, coldness of the limbs, and cyanosis were the most common symptoms upon admission. Loss of consciousness, ill and toxicity, apnea, cyanosis of limbs and mucous membranes, poor pulse, cold limb, and mydriasis were significantly correlated with death on admission [15].

Systolic and diastolic blood pressure and body temperature were meaningfully lower in the death group than in the rescue group and are consistent with other studies[16]. The history of neurological diseases has been recorded only in $13.95 \%$ of patients.

Paraclinical manifestations: Many paraclinical manifestations were observed during hospitalization. The most common manifestations were hyperglycemia, hypernatremia, hypokalemia, elevated BUN and $\mathrm{Cr}$, leukocytosis, increased PT and INR, and various $A B G$ disorders[17].

There was a meaningful difference in $\mathrm{Hb}, \mathrm{pH}$, and $\mathrm{HCO} 3$ between the two groups at the time of admission. No difference in $\mathrm{Hb}$ between the two rescue groups was observed in another study[18]. In this case, it is crucial to consider fast and undiagnosed gastrointestinal bleeding. Studies have shown that RBC pack cell transfusion can be powerful in improving the outcome[19].

A meaningful correlation was observed between leukocytosis, hyperglycemia, increased $\mathrm{Cr}$, increased PT, high $\mathrm{PCO} 2$, and low $\mathrm{HCO} 3$ with treatment outcomes, which was consistent with the results of other studies [15]. There was a wide range of ECG changes, similar to other studies [8]. Echocardiography showed weak contraction in one case and a drop in EF in another case. 
Treatment: The first measures in primary centers include gastric lavage with normal saline, administration of charcoal, ranitidine, and intravenous sodium bicarbonate. A controversy over gastric lavage with normal saline seems to be due to insufficient awareness of the medical community about this issue [20]. There was a meaningful correlation between ranitidine administration in primary centers and treatment outcomes. Ranitidine may diminish phosphine gas release by inhibiting gastric acid.

The most common measures taken on both dispatched and non-dispatched patients upon admission at the center have been intravenous sodium bicarbonate prescription, as well as prescribing magnesium sulfate, $\mathrm{n}$ acetylcysteine, oral sodium bicarbonate, vitamin $\mathrm{C}$, vitamin E, charcoal, and sorbitol [8].

The relationship between the administration of $\mathrm{N}$ acetylcysteine at admission and the consequences of treatment was significant. This finding was consistent with the results of some studies and inconsistent with the results of some other studies[21]. The administration of injectable bicarbonate at admission is correlated with the outcome of treatment. Bicarbonate is effective in correcting metabolic acidosis in ALP toxicity. This finding is consistent with the results of other studies.

Most measures taken after statistical treatment include infusion of bicarbonate, daily vitamins $C$ and $E$, ranitidine $\mathrm{BD}$, magnesium sulfate, a repeated dose of charcoal, and NAC. The first four treatments were meaningfully correlated to the rate of survival.

Novel treatments, hyperinsulinemia, oxime, hyperbaric oxygen, and hemodialysis have not been used. Only two cases of oral administration of vegetable oils have been recorded.

In general, combination therapy by simultaneous administration of active charcoal, ranitidine, injectable sodium bicarbonate, continuous infusion of bicarbonate, vitamins $\mathrm{E}$ and $\mathrm{C}$, and $\mathrm{N}$-acetylcysteine seem to be effective in treating patients with ALP toxicity.

Next attempts to suicide: There was no study to cover the next attempts to suicide in patients with ALP toxicity.

Out of 17 rescued patients accessible in this study, only one patient has attempted suicide again and $94.11 \%$ of patients had no intention to suicide again. Psychiatric counseling results showed that $91.66 \%$ of the counseled patients attempted suicide by impulse and without a previous plan.

Subsequent complications: Types of effusion (pleural effusion, ascites, and genital edema) and hematuria were among common secondary and late complications. Some types of edema and effusion are expected given the adverse effect of the toxicity on cell wall permeability, especially terminal capillary vessels [22]. Persistent epigastric burning was seen in one patient presumably due to thermal injury that may necessitate more supportive treatment of the gastric mucosa in the acute phase. Some herbal oils and extracts have been proposed [2] but their effectiveness remains unclear. In this study, only one patient was re-admitted because of a complaint about shortness of breath and the diagnosis of pneumonia or pleural effusion. Studies show that pulmonary edema and pneumonia can be among complications of ALP toxicity[23]

\section{Ethical considerations}

- Patients paid no for attending this study.

- No diagnostic or therapeutic action was taken while exerting adverse consequences on participants.

- All information obtained from the files of patients was used confidentially and privately.

- All the necessary ethical considerations were respected on call to preserve the dignity of participants.

Acknowledgment:: This study is a thesis of Malika Vaez Tehrani. It is financially supported by the vice-chancellor of research affairs of the Ahvaz Jundishapur University of Medical Sciences. The authors would like to thank the Clinical Research Development Unit of Razi Hospitals. Ethical approval was obtained from the Ethical Committee of Ahvaz Jundishapur University of Medical Sciences, and the thesis number is (GP95129)

Conflicts of Interest: The authors declare that they have no conflicts of interest

\section{REFERENCES}

[1] H. Hamade et al., "Human Zinc Phosphide Exposure in Lebanon: A Case Report and Review of the Literature," Clin. Pract. Cases Emerg. Med., vol. 5, no. 1, p. 50, Jan. 2021, doi: 10.5811/CPCEM.2020.10.47397.

[2] D. Yadav, R. Bhattacharyya, and D. Banerjee, "Acute aluminum phosphide poisoning: The menace of phosphine exposure," Clin. Chim. Acta, vol. 520, pp. 34-42, Sep. 2021, doi: 10.1016/J.CCA.2021.05.026.

[3] R. Ghimire et al., "Intentional pesticide poisoning and pesticide suicides in Nepal," Clin. Toxicol., 2021, doi: 10.1080/15563650.2021.1935993.

[4] A. FAIZ, K. K. MOHD, M. ASAD, and A. JAMAL, "Aluminum Phosphide Poisoning and Blast in Gastric Tube; A Case Report," vol. 8, no. 3. ASIA PACIFIC JOURNAL OF MEDICAL TOXICOLOGY (APJMT), pp. 101-103, Jan. 01, 2019, Accessed: Jul. 11, 2021. [Online]. Available: https://www.sid.ir/en/journal/ViewPaper.aspx?id=807341.

[5] Y. N, H. HMMTB, S. T, C. R, and K. A, "A case report of zinc phosphide poisoning: complicated by acute renal failure and tubulo interstitial nephritis.," BMC Pharmacol. Toxicol., vol. 18, no. 1, pp. 37-37, May 2017, doi: 10.1186/S40360-0170144-7.

[6] A. M. Shakur, N. A. Garba, I. Ahmadu, D. Apollos, M. O. Asani, and I. Aliyu, "Accidental poisoning with aluminum phosphide presenting with excessive cholinergic symptoms with response to atropine: A case report," Avicenna J. Med., vol. 11, no. 1, p. 58, 2021, doi: 10.4103/AJM.AJM 15620.

[7] B. Vazirianzadeh, S. Maraghi, M. S.- IJSS, and undefined 2017, "Evaluating some medical lab parameters among hospitalized children in Khuzestan following scorpion sting, SW Iran," researchgate.net, vol. |, 2017, doi: 10.17354/ijssJunel/2017/014.

[8] D. E. Bogale, B. D. Ejigu, and T. A. Muche, "Clinical Profile and Treatment Outcome of Aluminum Phosphide Poisoning in Felege Hiwot Referral Hospital, Northwest Ethiopia: A Retrospective Study," Open Access Emerg. Med., vol. 13, p. 239, Jun. 2021, doi: 10.2147/OAEM.S313181.

[9] A. Maleki et al., "Adjuvant potential of selegiline in treating acute toxicity of aluminium phosphide in rats," Basic Clin. Pharmacol. Toxicol., vol. 125, no. 1, pp. 62-74, Jul. 2019, doi: $10.1111 /$ BCPT.13207.

[10] "A Survey on the Pattern of Aluminum Phosphide Poisoning in Gorgan, North of Iran," Beyhagh, vol. 21, no. 4, pp. 55-64, Jun. 2017, Accessed: Jul. 13, 2021. [Online]. Available: http://beyhagh.medsab.ac.ir/article_939.html. 
[11] A. Shafahi, B. Mostafazadeh, and B. Dadpour, "Inhalational Toxicity of Aluminum Phosphide as an Ongoing Concern; a Report of Two Cases," Arch. Acad. Emerg. Med., vol. 7, no. 1, pp. e69-e69, Nov. 2019, doi: 10.22037/AAEM.V7I1.509.

[12] N. M. Emam, S. S. Hamed, D. Alsaied, and M. Ahmed, "COULD N. ACETYL CYSTEINE PROLONG SURVIVAL TIME IN ACUTE ALUMINUM PHOSPHIDE POISONING AMONG EGYPTIAN PATIENTS?," Orig. Artic. NAC Prolong. Surviv. Time Acute Alp Poisoning.... ESCTJ, vol. 8, no. 2, 2020.

[13] O. Mehrpour et al., "Epidemiological and clinical profiles of acute poisoning in patients admitted to the intensive care unit in eastern Iran (2010 to 2017)," BMC Emerg. Med. 2018 181, vol. 18, no. 1, pp. 1-9, Sep. 2018, doi: 10.1186/S12873-018-0181-6.

[14] S. Hallaj, A. B. Mohammadi, A. Ghorbani, A. Ostadi, and M. Z. Nahandi, "Case report: Successful management of an aluminum phosphide poisoned patient following ventricular tachycardia," Int. J. Med. Toxicol. Forensic Med., vol. 10, no. 4, Nov. 2020, doi: 10.32598/IJMTFM.V1014.30473.

[15] M. Majidi, M. Jamalpour, and S. Nekoueifard, "The Prognostic Factors of Aluminum Phosphide Poisoning in Urmia: A-five-years Cross-sectional Study:," Int. J. Med. Toxicol. Forensic Med., vol. 11, no. 2, pp. 32663-32663, Jun. 2021, doi: 10.32598/IJMTFM.V11I2.32663.

[16] A. K. Pannu, A. Bhalla, A. Sharma, and N. Sharma, "'PGI Score': A Simplified Three-point Prognostic Score for Acute Aluminum Phosphide Poisoning," Indian J. Crit. Care Med., vol. 24, no. 9, p. 790, Oct. 2020, doi: 10.5005/JP JOURNALS-10071-23555.
[17] A. Ghodsi, B. Dadpour, and Z. S. Toroghi, "Aluminum phosphide poisoning, an unusual presentation," J Bas Res Med Sci, vol. 7, no. 3, pp. 71-74, 2020.

[18] N. Rahimi et al., "Fresh red blood cells transfusion protects against aluminum phosphide-induced metabolic acidosis and mortality in rats," 2018, doi: 10.1371/journal.pone.0193991.

[19] P. Kashefi, A. Rahmani, and M. Khalifesoltani, "Changes in the hemoglobin level after one unit of packed red blood cell transfusion in Intensive Care Unit patients," J. Res. Med. Sci., vol. 23, no. 1, p. 85, 2018, doi: 10.4103/JRMS.JRMS_64_17.

[20] A. M. Shakur, N. A. Garba, I. Ahmadu, D. Apollos, M. O. Asani, and I. Aliyu, "Accidental poisoning with aluminum phosphide presenting with excessive cholinergic symptoms with response to atropine: A case report," Avicenna J. Med., vol. 11, no. 1, p. 58, 2021, doi: 10.4103/AJM.AJM 15620.

[21] A. Karimani et al., "Antidotes for aluminum phosphide poisoning - An update," Toxicol. Reports, vol. 5, pp. 10531059, Jan. 2018, doi: 10.1016/J.TOXREP.2018.10.009.

[22] F. Nourbakhsh, S. Barangi, B. Dadpour, and S. H. Tajoddini, "Aluminum Phosphide Poisoning, Mechanism of Action and Treatment: a Literature Review," J. Kerman Univ. Med. Sci., vol. 26, no. 5, pp. 406-419, 2019, Accessed: Jul. 14, 2021. [Online]. Available: http://www.efsa.europa.eu/.

[23] G. Bilics et al., "Successful management of zinc phosphide poisoning-a Hungarian case," Int. J. Emerg. Med. 2020 131, vol. 13, no. 1, pp. 1-9, Sep. 2020, doi: 10.1186/S12245-020-00307-8. 